\title{
Sperm protein 17 is expressed in human nervous system tumours
} Fabio Grizzi 1,4, Paolo Gaetani² ${ }^{2}$ Barbara Franceschini1,4, Antonio Di Ieva², Piergiuseppe Colombo ${ }^{3}$, Giorgia Ceva-Grimaldi ${ }^{1,4}$, Angelo Bollati ${ }^{5}$, Eldo E Frezza 6 , E Cobos ${ }^{7}$ Riccardo Rodriguez y Baena ${ }^{2}$, Nicola Dioguardi ${ }^{1,4}$ and Maurizio Chiriva-Internati*8

\author{
Address: ${ }^{1}$ Scientific Direction, Istituto Clinico Humanitas, IRCCS, 20089 Rozzano, Milan, Italy, ${ }^{2}$ Department of Neurosurgery, Istituto Clinico \\ Humanitas, IRCCS, 20089 Rozzano, Milan, Italy, ${ }^{3}$ Department of Pathology, Istituto Clinico Humanitas, IRCCS, 20089 Rozzano, Milan, Italy, \\ 4 "Michele Rodriguez" Foundation. Scientific Institute for Quantitative Measures in Medicine, 20100 Milan, Italy, ${ }^{5}$ Department of Neurosurgery, \\ University of Brescia, Spedali Civili, Italy, ${ }^{6}$ Department of Surgery, Texas Tech University Health Sciences Center and Southwest Cancer Treatment \\ and Research Center, 79430 Lubbock, Texas, USA, ${ }^{7}$ Department of Internal Medicine, Texas Tech University Health Sciences Center and Southwest \\ Cancer Treatment and Research Center, 79430 Lubbock, Texas, USA and ${ }^{8}$ Department of Microbiology and Immunology, Texas Tech University \\ Health Sciences Center and Southwest Cancer Treatment and Research Center, 79430 Lubbock, Texas, USA \\ Email: Fabio Grizzi - fabio.grizzi@humanitas.it; Paolo Gaetani - paolo.gaetani@humanitas.it; \\ Barbara Franceschini - barbara.franceschini@humanitas.it; Antonio Di Ieva - diieva@hotmail.com; \\ Piergiuseppe Colombo - piergiuseppe.colombo@humanitas.it; Giorgia Ceva-Grimaldi - giorgia.cevagrimaldi@humanitas.it; \\ Angelo Bollati - bollati@med.unibs.it; Eldo E Frezza - eldo.frezza@ttuhsc.edu; E Cobos - everardo.cobos@ttuhsc.edu; Riccardo Rodriguez \\ y Baena - riccardo.rodriguez@humanitas.it; Nicola Dioguardi - nicola.dioguardi@humanitas.it; Maurizio Chiriva- \\ Internati* - maurizio.chirivainternati@ttuhsc.edu \\ * Corresponding author
}

Published: 26 January 2006

BMC Cancer 2006, 6:23 doi:10.1/86/I47|-2407-6-23
Received: 13 June 2005

Accepted: 26 January 2006

This article is available from: http://www.biomedcentral.com/I47I-2407/6/23

(c) 2006 Grizzi et al; licensee BioMed Central Ltd.

This is an Open Access article distributed under the terms of the Creative Commons Attribution License (http://creativecommons.org/licenses/by/2.0), which permits unrestricted use, distribution, and reproduction in any medium, provided the original work is properly cited.

\begin{abstract}
Background: Human sperm protein 17 (Spl7) is a highly conserved protein that was originally isolated from a rabbit epididymal sperm membrane and testis membrane pellet. It has recently been included in the cancer/testis (CT) antigen family, and shown to be expressed in multiple myeloma and ovarian cancer. We investigated its immunolocalisation in specimens of nervous system (NS) malignancies, in order to establish its usefulness as a target for tumour-vaccine strategies.

Methods: The expression of SpI7 was assessed by means of a standardised immunohistochemical procedure [(mAb/antigen) MFI/SpI7] in formalin-fixed and paraffin embedded surgical specimens of NS malignancies, including 28 neuroectodermal primary tumours $(6$ astrocytomas, 16 glioblastoma multiforme, 5 oligodendrogliomas, and I ependymoma), 25 meningeal tumours, and five peripheral nerve sheath tumours (4 schwannomas, and I neurofibroma),.

Results: A number of neuroectodermal (21\%) and meningeal tumours (4\%) were found heterogeneously immunopositive for Sp 17. None of the peripheral nerve sheath tumours was immunopositive for Sp 17. The expression pattern was heterogeneous in all of the positive samples, and did not correlate with the degree of malignancy.

Conclusion: The frequency of expression and non-uniform cell distribution of Sp I7 suggest that it cannot be used as a unique immunotherapeutic target in NS cancer. However, our results do show the immunolocalisation of Sp 17 in a proportion of NS tumour cells, but not in their non-pathological counterparts. The emerging complex function of Spl7 makes further studies necessary to clarify the link between it and immunopositive cells.
\end{abstract}




\section{Background}

Brain and other nervous system (NS) tumours are a group of neoplasms that vary in terms of their site of origin, morphological features, genetic alterations, growth potential, invasiveness, tendency to progression and recurrence, and response to treatments [1-4]. Their still highly unfavourable prognosis underlines the need for new therapeutic approaches, one of the most attractive of which is targeted immunotherapy (any approach aimed at mobilising or manipulating a patient's immune system to treat or cure disease), which is widely considered to be more specific and less toxic than conventional surgery, chemotherapy or radiation therapy $[5,6]$. However, although the bloodbrain barrier and the claimed immunological privilege of the brain are not necessarily obstacles to effective brain immunotherapy, these strategies are currently limited by the paucity of cloned NS target antigens and the fact that our understanding of anti-tumour immune responses in the NS is frequently extrapolated from other tissues having little in common with it $[7,8]$.

A cancer-testis (CT) family of tumour-associated antigens has been identified and their encoding genes extensively investigated [9-13]. Their immunogenicity and restricted expression in normal tissues makes them ideal molecules for immunotherapeutic procedures $[9,10]$.

Sperm protein 17 (Sp17) has recently been entitled as novel CT in ovarian cancer, multiple myeloma and other blood malignancies [14-16], and mRNA encoding Sp17 has been found in two myeloma cell lines, $17 \%$ of a series of patients with multiple myeloma, and in primary tumour cells from $70 \%$ of a series of patients with ovarian carcinoma $[14,15]$. At protein level, Sp17 has been found in human germinal cells of the testis other than spermatogonia [17], as well as in the ciliated epithelia of the respiratory airways and both male and female reproductive systems [18]. It has also recently been identified in the synoviocytes of females with rheumatoid arthritis [19], and in the melanophages of cutaneous melanocytic lesions [20]. Although these findings have all raised the question as to whether Sp17 may be a useful target for tumour immunotherapy [21-23], they also highlight the fact that its tissue distribution in humans is more complex than originally thought.

The expression frequencies of many CT antigens have been determined in a variety of neoplasms [11], but little is known about their expression in human NS tumours.

The aim of this immunohistochemical study was to investigate the frequency of Sp17 expression at protein level and its cell pattern distribution in various histological subtypes of human NS tumours, including neuroectodermal, meningeal and nerve sheath lesions.

\section{Methods \\ Patients}

The study was conducted in accordance with the guidelines of the Ethics Committee of the hospital treating the patients (Istituto Clinico Humanitas, Rozzano, Milan, Italy), all of whom were fully informed of the possible discomfort and risks of surgical treatment.

All of the analysed tissues were taken from patients admitted to the Istituto Clinico Humanitas, Rozzano, Milan, between 2002 and 2004. The patients' mean age was 58 years (range: 18-79), and the group consisted of 26 males and 32 females.

\section{Tissue specimens}

The NS tumour specimens represented 28 neuroectodermal tumours ( 6 astrocytomas, 16 glioblastomas, 5 oligodendrogliomas, and 1 ependymoma), 25 meningeal tumours, and five nerve sheath tumours ( 4 schwannomas, 1 neurofibroma).

To evaluate whether Sp17 is expressed at protein level in non-pathological NS tissue, three further specimens were taken from one male and two female patients (mean age $58 \pm 15$ years) who underwent surgical resection for neoplastic disease and were sampled at the periphery of the lesions. The absence of cell or tissue pathologies was subsequently confirmed by microscopy.

All of the specimens were fixed in 10\% neutral buffered formalin ( 12 hours) and embedded in paraffin.

\section{Reverse transcription-polymerase chain reaction (RT- PCR) amplification of Sp I 7 mRNA}

A panel of normal tissue total RNA was obtained from Strategene Corp. (La Jolla, CA). All of the RNAs were first treated with DNAse I (Ambion, Austin, TX) to remove genomic DNA contamination, and first- strand cDNA was synthesised from $1 \mu \mathrm{g}$ by means of random hexamer primers. The primers for 5' Sp17 PCR was 5'-GGCAGTTCTTACCAAGAAGAT-3', and that for 3' Sp17 PCR was 5'GGAGGTAAAACCAGTGTCCTC-3', both of which amplify a complementary DNA (cDNA) of approximately 500 base-pairs. PCR was performed by means of 35 amplification cycles at an annealing temperature of $55^{\circ} \mathrm{C}$. The positive control amplification contained a plasmid with the Sp17 cDNA and a negative control of the PCR reaction mixture except for the substitution of cDNA by water. RNA integrity in each sample was checked by amplifying a $\beta$-actin gene segment. The successful removal of DNA contamination was confirmed by amplifying the RNA in each sample without the reverse transcription reaction. The PCR products were visualised on an ethidium bromide agarose gel for a DNA band of the expected size. All 
Table I: Patient baseline characteristics.

\begin{tabular}{lccc}
\hline Tumour histological subtype & Patients (n) & Age* (years) & Sex \\
\hline Astrocytoma & 6 & $60 \pm 20$ & $4 \mathrm{M} / 2 \mathrm{~F}$ \\
Oligodendroglioma & 5 & $45 \pm 10$ & $5 \mathrm{M}$ \\
Ependymoma & 1 & 22 & $\mathrm{IF}$ \\
Glioblastoma & 16 & $59 \pm 17$ & $5 \mathrm{M} / \mathrm{IIF}$ \\
Meningioma & 25 & $60 \pm 14$ & $8 \mathrm{M} / \mathrm{I7F}$ \\
Swhanoma & 4 & $65 \pm 9$ & $3 \mathrm{M} / \mathrm{IF}$ \\
Neurofibroma & 1 & 32 & $\mathrm{IM}$
\end{tabular}

*Data are expressed as mean \pm standard deviation

of the results were confirmed by two independent RTPCRs.

\section{Immunohistochemistry}

Two- $\mu \mathrm{m}$ thick sections were cut and processed for immunohistochemistry. After deparaffining and rehydration, the sections were immersed in a bath (Dako, Milan, Italy) for antigen retrieval for $45 \mathrm{~min}$ at $98^{\circ} \mathrm{C}$ in a freshly made EDTA $1 \mathrm{mM}$ solution, incubated with $3 \% \mathrm{H}_{2} \mathrm{O}_{2}$ for 15 min, and then treated with primary antibodies raised against Sp17 [monoclonal murine anti-Sp17 (Sp17MF1), dilution 1:100 in phosphate buffered saline, PBS] at room temperature for two hours, or with $1 \mathrm{mg} / \mathrm{ml}$ mouse IgG1 (Dako, Milan, Italy) as a negative control. This was followed by 30 min incubation with the DAKO Envision system (Dako, Milan, Italy). 3,3'-diaminobenzidine tetrahydrochloride (Sigma Ltd, Missouri, USA) was used as a chromogen to yield brown reaction products.

The testis specificity of the binding of the Sp17 antibodies has been confirmed in competitive binding assays showing the abrogation of testicular staining if the Sp17 antibodies are pre-incubated with a very high concentration $(100 \mu \mathrm{g} / \mathrm{ml})$ of soluble Sp17 recombinant protein [24].

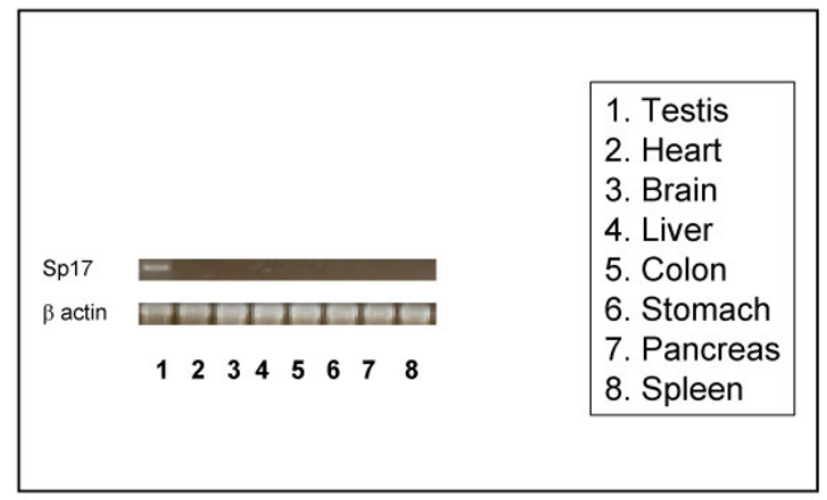

Figure I

RT-PCR showing SpI7 transcripts in testis, but not in brain, colon, heart, liver, stomach, pancreas, and spleen (a). Control amplification for the $\beta$-actin gene segment (b).
The specificity of the primary antibodies raised against Sp17 was also shown by Western blotting analyses $[17,18,25]$.

The nuclei were lightly counterstained with hematoxylin solution (Medite, Bergamo, Italy).

The immunohistochemical reaction was observed using a light microscope (Leica DMLA, Italy) at $40 \times$ and $100 \times$ objective magnifications, and was semi-quantitatively graded into four classes by two expert neuropathologists on the basis of the frequency of Sp17 in NS cancer cells: negative $=$ no immunopositive cells $(x) ;+=$ low frequency $(x \leq 25 \%) ;++=$ moderate frequency $(25 \%<x \leq 50 \%) ;+++$ $=$ high frequency $(50 \%<x \leq 75 \%) ;++++=$ very high frequency $(75 \%<x \leq 100 \%)$.

\section{Statistical analysis}

All of the data are expressed as mean values \pm standard deviation. The results were analysed using Statistica software (StatSoft Inc., Tulsa, OK, USA). Univariate analysis was performed by means of the Student $t$ or chi-square test as required for parametric and categorical variables. A $p$ value of less than 0.05 was considered to be statistically significant.

\section{Results \\ Patient baseline characteristics}

Table 1 shows the investigated NS lesions and the patients' baseline characteristics. Despite the limited number of cases, the mean age of the patients with oligodendrogliomas ( $45 \pm 10$ years) was significantly different from that of those with meningiomas $(60 \pm 14$ years; $\mathrm{p}=0.03)$ or schwannomas $(65 \pm 9$ years; $\mathrm{p}=0.01)$. There was no significance difference in gender distribution between the neoplastic histological subtypes.

\section{RT-PCR amplification of SpI7 mRNA}

Using a pair of sequence-specific primers, mRNA encoding Sp17 was found in testis, but not in brain, colon, heart, liver, stomach, pancreas, and spleen (Fig. 1). 


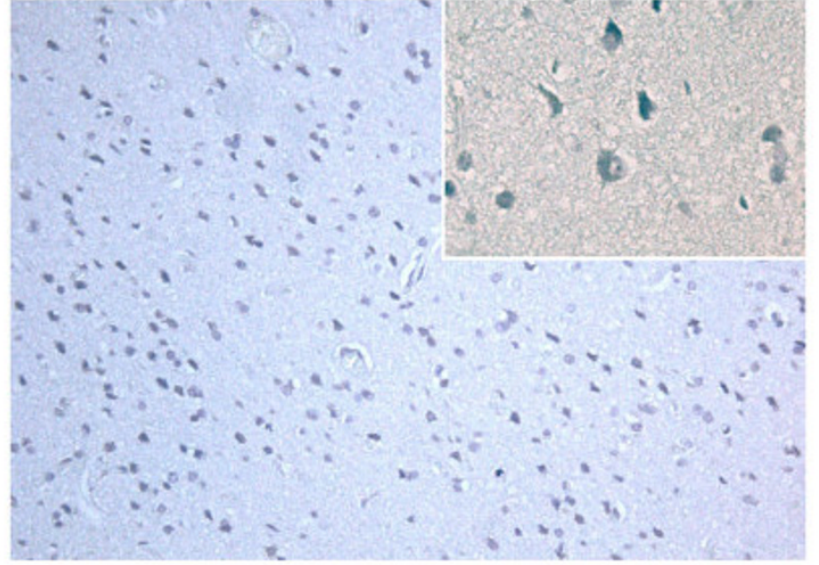

\section{Figure 2}

Immunohistochemistry of SpI7 in non-pathological nervous system tissues. There were no immunopositive cells (original magnification, $20 \times$, $40 \times$ inset).

\section{Immunohistochemistry}

Table 2 shows the frequencies of Sp17 expression at protein level. All three non-pathological NS specimens were immunonegative (Fig. 2), but Sp17 was found in 21\% of the neuroectodermal and $4 \%$ of the meningeal lesions. Excluding the only ependymoma (which was immunopositive), the highest frequency was among the astrocytomas. None of the nerve sheath tumours was immunopositive. In all of the immunopositive lesions, Sp17 was localised in the cytoplasm of a subset of cancer cells. Each immunopositive case was semi-quantitatively graded by two expert neuropathologists (Tab. 3). The expression pattern was always heterogeneous (Fig. 3) and did not correlate with the degree of malignancy.

Sp17 was expressed in six female patients, who accounted for $86 \%$ of the immunopositive patients and one male. Considering the presumed cell of origin of the lesions, Sp17 was found in two females (100\%) and no males with astrocytoma; two females (18\%) and one male (20\%) with glioblastoma; one female (5.8\%) and no males with meningioma; and one female (100\%) with ependymoma.

There was no difference in the mean age of the patients who were Sp17 immunopositive or immunonegative.

\section{Discussion}

Despite advances in surgical techniques, imaging methodologies and adjuvant radiation therapy, the unfavourable prognosis of most brain and other NS tumours [1-3] has prompted an intensive search for effective treatment alternatives. Novel immunostimulatory strategies, such as immunogenic therapy, directed cytokine delivery and
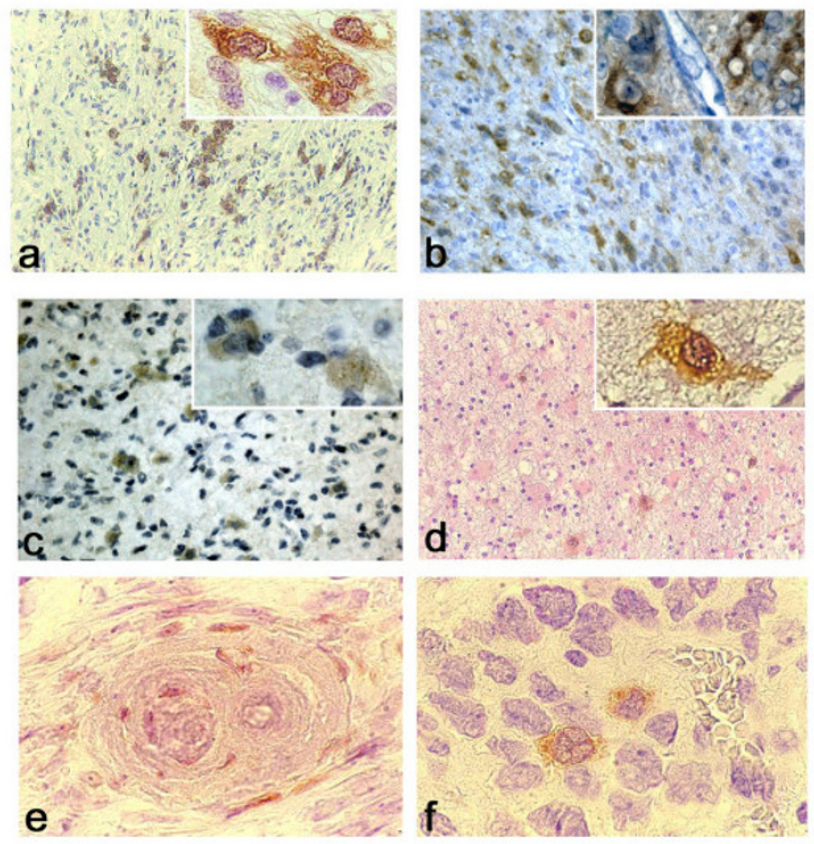

\section{Figure 3}

Immunohistochemistry of Spl7 in nervous system neoplasms: $a-c$. glioblastomas; $d$. astrocytoma; e. meningioma; $f$. ependimoma. In all cases, Sp 17 was localised in the cytoplasm of a few isolated and scattered tumoral cells. (a-d, 40x original magnification, $100 \times$ insets; e-f, $100 \times$ original magnification).

dendritic cell manipulation, have recently been used in patients with malignant NS tumours in an attempt to improve the results obtained with surgery, chemotherapy and radiotherapy $[7,8]$.

Since the first cloning of a human tumour antigen, melanoma antigen-1 (MAGE-1), the identification of tumour antigens capable of inducing an immune response in cancer patients, and the development of vaccines targeting these antigens, have been formidable tasks confronting tumour immunologists [5,6,9-13]. It has long been accepted that immunotherapy is a powerful cancer treatment, but it has been mainly limited by a poor understanding of the complexity of human beings, the immune system, and highly intricate host-tumour interactions $[23,26]$.

The introduction of T-cell epitope cloning and, subsequently, a method based on the serological analysis of recombinant tumour cDNA expression libraries using autologous serum (SEREX), has led to the identification of almost 44 CT gene families [10,11]. Sp17 has recently been included among them because, although it was originally thought to be gamete-specific, it has also been iden- 
Table 2: Sperm protein 17 expression frequencies in different histological subtypes of primary nervous system neoplasms. The lesions are categorised on the basis of their presumed cell of origin.

\begin{tabular}{lc}
\hline \multicolumn{1}{c}{ Cell of origin } & number positive/total number tested \\
\hline Glial cell & \\
Astrocytoma & $2 / 6$ \\
Oligodendroglyoma & $0 / 5$ \\
Ependymoma & $1 / 1$ \\
Glioblastoma & $3 / 16$ \\
\hline Arachinodal cell & \\
Meningioma & $1 / 25$ \\
\hline Nerve Sheath cell & \\
Swhanoma & \\
Neurofibroma & $0 / 4$ \\
\end{tabular}

tified in various human somatic and tumoral tissues [1416,18-20,27-29]. The expression frequencies of many CT antigens have been determined in many neoplasms, but little is known about their expression in human NS tumours

We show that Sp17 is heterogeneously expressed at protein level in a proportion of neuroectodermal and meningeal tumours, but not in nerve sheath neoplasms or nonpathological NS tissues.

Sahin et al. (2000) have recently investigated the expression of seven CT genes (MAGE-3, NY-ESO-1, HOM-MEL40/SSX-2, SSX-1, SSX-4, HOM-TES-14/SCP-1, and HOMTES-85) in a series of human brain tumour specimens, and concluded that a majority of oligoastrocytomas and astrocytomas might be amenable to specific immunotherapeutic interventions, although the identification of additional tumour-specific antigens frequently expressed in gliomas should allow for the development of widely applicable polyvalent glioma vaccines [30].

However, it must be pointed out that the authors investigated mRNA expression, which does not necessarily correlate directly with protein expression. The discrepancies between the frequencies of gene and protein expression may reflect variations in tissue sampling, the heterogeneity tumour cells, or differences in the sensitivity of detection $[10,11,26,31]$. The expression of CT antigens in clinical material has mainly been studied by RT-PCR at gene expression level, but this approach is partially limited by the fact that it does not allow the quantification of cancer cells positive for CT antigens [32]. On the contrary, the availability of specific antibodies enables the recognition of candidate antigens in examined tissues, thus highlighting both the number and type of cells that are
Table 3: Semi-quantitative grading of Sp 17 expression in NS tumours.

\begin{tabular}{lcc}
\hline Sample & Immunohistochemistry & Pattern \\
\hline Astrocytoma I & +++ & Cyto \\
Astrocytoma 2 & +++ & Cyto \\
Ependymoma & ++ & Cyto \\
Glioblastoma I & ++ & Cyto \\
Glioblastoma 2 & +++ & Cyto \\
Glioblastoma 3 & +++ & Cyto \\
Meningioma & + & Cyto \\
\hline
\end{tabular}

Notes: $+=$ low frequency $(x \leq 25 \%)$ of cells immunopositive for SpI $7 ;++=$ moderate frequency $(25 \%<x \leq 50 \%)$ of cells immunopositive for Sp I 7 ; +++ = high frequency $(50 \%<x \leq 75 \%)$ of cells immunopositive for Sp I7; ++++ = very high frequency of cells immunopositive for SpI7 (75\% < x $\leq 100 \%)$. Cyto = cytoplasmic SpI7 localization.

immunopositive for a given antigen $[11,23,32]$; however, the number of monoclonal antibodies against CT proteins that can be used in immunohistochemical or biochemical analyses of protein expression is still relatively limited $[11,32]$.

On the basis of our findings, it can be said that:

a. Sp17 can be recognised at protein level in various neuroectodermal and meningeal tumours, but not in nerve sheath tumours.

b. Sp17 is localised in the cytoplasm of a variable number of tumoral cells (Fig. 3). The focal expression of Sp17 in NS cancer cells suggests that this antigen might not be sufficient as a unique target for immunotherapeutic approaches. It is now widely accepted that a prerequisite for the success of any tumour-specific therapeutic strategy is the existence and identification of antigen targets that are not only exclusively or preferentially expressed in malignant tissues, but also expressed in a high percentage of neoplastic cells in a large number of patients [10$12,23,31,33]$.

$c$. Although the clinical and patient characteristics of our study population were similar to those described in other series, its redistribution on the basis of immunohistochemical findings highlights the fact that $86 \%$ of the samples immunopositive for $\mathrm{Sp} 17$ were taken from female patients. Although this finding needs to be confirmed in a large cohort of patients, it extends those of a previous study by Takeoka et al. (2000), who found Sp17 expressed in the synoviocytes of females with rheumatoid arthritis [19].

The variable frequency of the expression of CT antigens, and their uneven distribution in cancer cells [34], have been recently been attributed to the spatial and temporal 
complexity of tumoral lesions and their non-linear interactions with the body environment [26,35-37]. These basic characteristics have led to the development of a system-level approach that is not only intended to generate a human cancer map of the expression of candidate antigens, but also to highlight common features underlying tumours of unrelated histological origin, thus improving our basic knowledge of tumour complexity [21].

Our study is the first to show the expression of Sp17 at protein level in a proportion of NS tumours but not in non-pathological NS tissues. This extends our knowledge of CT antigens and NS cancer [38-41], and reinforces the need for further studies aimed at clarifying the complex function of Sp17, which clearly seems to go beyond the simple role in the fertilisation process that was originally attributed to it.

\section{Competing interests}

The author(s) declare that they have no competing interests.

\section{Authors' contributions}

FG, MCI conceived, coordinated and designed the study, and drafted the manuscript; PG, BF, ADI, PC, GCG, AB, $\mathrm{EEF}, \mathrm{EC}, \mathrm{RRB}, \mathrm{ND}$ participated in designing the study and drafting the manuscript. All of the authors have read and approved the final manuscript.

\section{References}

I. Cher $M$ : Cancer and the nervous system. MJA 200I, I 75:277-282.

2. Gurney JG, Kadan-Lottick N: Brain and other central nervous system tumors: rates, trends, and epidemiology. Curr Opinion Oncol 2001, 13:160-166.

3. Legler JM, Ries LA, Smith MA, Warren JL, Heineman EF, Kaplan RS, Linet MS: Cancer surveillance series [corrected]: brain and other central nervous system cancers: recent trends in incidence and mortality. J Natl Cancer Inst 1999, 9 I: | 382- I390.

4. Noble M, Dietrich J: The complex identity of brain tumors: emerging concerns regarding origin, diversity and plasticity. Trends Neurosci 2004, 27: I48-I54.

5. Gilboa E: The promise of cancer vaccines. Nat Rev Cancer 2004, 4:40I-4II.

6. Steinman RM, Mellman I: Immunotherapy: bewitched, bothered, and bewildered no more. Science 2004, 305:197-200.

7. Ehtesham M, Black KL, Yu JS: Recent progress in immunotherapy for malignant glioma: treatment strategies and results from clinical trials. Cancer Control 2004, I I: | 92-207.

8. Fenstermaker RA, Ciesielski MJ: Immunotherapeutic strategies for malignant glioma. Cancer Control 2004, I I:|8|-191.

9. Scanlan MJ, Gure AO, Jungbluth AA, Old LJ, Chen YT: Cancer/testis antigens: an expanding family of targets for cancer immunotherapy. Immunol Rev 2002, 188:22-32.

10. Lewis JD, Reilly BD, Bright RK: Tumor-associated antigens: from discovery to immunity. Int Rev Immunol 2003, 22:8I-I I 2.

II. Scanlan MJ, Simpson AJ, Old LJ: The cancer/testis genes: review, standardization, and commentary. Cancer Immun 2004, 4: I- I 5 .

12. Simpson AJG, Caballero OL, Jungbluth A, Chen Y-T, Old LJ: Cancertestis antigens: gametogenesis and cancer. Nat Rev Cancer 2005, 5:615-625.

13. Old LJ: Cancer/testis (CT) antigens: a new link between gametogenesis and cancer. Cancer Immun 200 I, I: I.
14. Chiriva-Internati M, Wang Z, Salati E, Timmins P, Lim SH: Tumor vaccine for ovarian carcinoma targeting sperm protein 17. Cancer 2002, 94:2447-2453.

15. Lim SH, Wang Z, Chiriva-Internati M, Xue Y: Sperm protein 17 is a novel cancer-testis antigen in multiple myeloma. Blood 2001, 97:1508-1510.

16. Lim SH, Bumm K, Chiriva-Internati M, Xue Y, Wang Z: MAGE-CI (CT7) gene expression in multiple myeloma: relationship to sperm protein I7. Eur J Haematol 2001, 67:332-334.

17. Grizzi F, Chiriva-Internati M, Franceschini B, Hermonat PL, Soda G, Lim SH, Dioguardi N: Immunolocalisation of sperm protein I7 in human testis and ejaculated spermatozoa. J Histochem Cytochem 2003, 9:1245-1248.

18. Grizzi F, Chiriva-Internati M, Franceschini B, Bumm K, Colombo P, Ciccarelli M, Donetti E, Gagliano N, Hermonat PL, Bright RK, Gioia $M$, Dioguardi N, Kast WM: Sperm protein 17 is expressed in human somatic ciliated epithelia. J Histochem Cytochem 2004, 52:549-554.

19. Takeoka Y, Kenny TP, Yago H, Naiki M, Gershwin ME, Robbins DL: Developmental considerations of sperm protein 17 gene expression in rheumatoid arthritis synoviocytes. Dev Immunol 2002, 9:97-102.

20. Franceschini B, Grizzi F, Colombo P, Soda G, Bumm K, Hermonat PL, Monti M, Dioguardi N, Chiriva-Internati M: Expression of human sperm protein 17 in melanophages of cutaneous melanocytic lesions. Br J Dermatol 2004, I 50:780-782.

21. Chiriva-Internati M, Grizzi F, Franceschini B, Hermonat PL, Bright RK, Bumm K, Dioguardi N, Kast WM: Is sperm protein I 7 a useful target for tumor immunotherapy? Blood 2003, 1 02:2308-2309.

22. Grizzi F, Franceschini B, Hermonat PL, Liu Y, Chiriva-Internati M: Some remarks on the somatic expression of sperm protein I 7. Int J Cancer 2004, I I I:972-773.

23. Chiriva-Internati M, Grizzi F, Bright RK, Kast WM: Cancer immunotherapy: avoiding the road to perdition. J Transl Med 2004, 2:26.

24. Chiriva-Internati M, Wang Z, Xue Y, Bumm K, Hahn AB, Lim SH: Sperm protein 17 (Sp I 7) in multiple myeloma: Opportunity for myeloma-specific donor $T$ cell infusion to enhance graftversus-myeloma effect without increasing graft-versus-host disease risk. Eur J Immunol 200 I, 3 I :2277-2283.

25. Grizzi F, Lim SH, Chiriva-Internati M, Franceschini B, Wang Z, Lawrence $D$, Dioguardi $N$ : Sperm protein 17 is not expressed on normal leukocytes. Blood 2002, 99:3479-3480.

26. Marincola FM, Wang E, Herlyn M, Seliger B, Ferrone S: Tumors as elusive targets of $\mathbf{T}$-cell-based active immunotherapy. Trends Immunol 2003, 24:335-442.

27. Straughn JM Jr, Shaw DR, Guerrero A, Bhoola SM, Racelis A, Wang Z, Chiriva-Internati M, Grizzle WE, Alvarez RD, Lim SH, Strong TV: Expression of sperm protein 17 (Sp I 7) in ovarian cancer. Int J Cancer 2004, I08:805-8II.

28. Arora S, Matta A, Shukla NK, Deo SV, Ralhan R: Identification of differentially expressed genes in oral squamous cell carcinoma. Mol Carcinog 2005, 42:97-108.

29. Ostrowsky LE, Blackburn K, Radde KM, Moyer MB, Schlatzer DM, Moseley A, Boucher RC: A proteomic analysis of human cilia. Identification of novel components. Mol Cell Proteomics 2002, I:45 I -465.

30. Sahin U, Koslowski M, Tureci O, Eberle T, Zwick C, Romeike B, Moringlane JR, Schwechheimer K, Feiden W, Pfreundschuh M: Expression of cancer testis genes in human brain tumors. Clin Cancer Res 2000, 6:3916-3922.

31. Lim SH, Periman P, Klug P, Weidanz J, Whitton V, Chiriva-Internati $M$, Wang $Z$, Wright S: Defining tumor antigens: mRNA, protein or cytotoxicity? Trends Immunol 2002, 23:236-237.

32. Juretic A, Spagnoli GC, Schultz-Thater E, Sarcevic B: Cancer/testis tumour-associated antigens: immunohistochemical detection with monoclonal antibodies. Lancet Oncol 2003, 4: 104-109.

33. Schultze JL, Vonderheide RH: From cancer genomics to cancer immunotherapy: toward second-generation tumor antigens. Trends Immunol 200I, 22:516-523.

34. Jungbluth AA, Stockert E, Chen YT, Kolb D, Iversen K, Coplan K, Williamson B, Altorki N, Busam KJ, Old LJ: Monoclonal antibody MA454 reveals a heterogeneous expression pattern of MAGE-I antigen in formalin-fixed paraffin embedded lung tumours. Br J Cancer 2000, 83:493-497. 
35. Grizzi F, Chiriva-Internati M: The complexity of anatomical systems. Theor Biol Med Model 2005, 2:26.

36. Kitano $\mathrm{H}$ : Cancer as a robust system: implications for anticancer therapy. Nat Rev Cancer 2004, 4:227-235.

37. Ransohoff DF: Rules of evidence for cancer molecular marker discovery and validation. Nat Rev Cancer 2004, 4:309-3I4.

38. Bodey B, Siegel SE, Kaiser HE: MAGE-I, a cancer/testis-antigen, expression in childhood astrocytomas as an indicator of tumor progression. In Vivo 2002, 16:583-588.

39. Mintz A, Debinski W: Cancer genetics/epigenetics and the $\mathbf{X}$ chromosome: possible new links for malignant glioma pathogenesis and immune-based therapies. Crit Rev Oncog 2000, II:77-95.

40. Debinski W, Gibo DM: Molecular expression analysis of restrictive receptor for interleukin 13, a brain tumor-associated cancer/testis antigen. Mol Med 2000, 6:440-449.

4I. Schmits R, Cochlovius B, Treitz G, Regitz E, Ketter R, Preuss KD, Romeike BF, Pfreundschuh M: Analysis of the antibody repertoire of astrocytoma patients against antigens expressed by gliomas. Int J Cancer 2002, 98:73-77.

\section{Pre-publication history}

The pre-publication history for this paper can be accessed here:

http://www.biomedcentral.com/1471-2407/6/23/prepub

Publish with Biomed Central and every scientist can read your work free of charge

"BioMed Central will be the most significant development for disseminating the results of biomedical research in our lifetime. "

Sir Paul Nurse, Cancer Research UK

Your research papers will be:

- available free of charge to the entire biomedical community

- peer reviewed and published immediately upon acceptance

- cited in PubMed and archived on PubMed Central

- yours - you keep the copyright 